\title{
Existence of positive periodic solutions for abstract evolution equations
}

\section{Qiang $\mathrm{Li}^{*}$ and Yongxiang $\mathrm{Li}$}

"Correspondence:

Iznwnuliqiang@126.com

Department of Mathematics,

Northwest Normal University,

Lanzhou, 730070, People's Republic of China

\begin{abstract}
In this paper, we discuss the existence of the positive time periodic mild solutions for the evolution equation in an ordered Banach space $E, u^{\prime}(t)+A u(t)=f(t, u(t)), t \in \mathbb{R}$, where $A: D(A) \subset E \rightarrow E$ is a closed linear operator and $-A$ generates a positive compact semigroup $T(t)(t \geq 0)$ in $E$, the nonlinear function $f: \mathbb{R} \times E \rightarrow E$ is continuous and $f(t, x)$ is $\omega$-periodic in $t$. We apply the operator semigroup theory and the Leray-Schauder fixed point theorem to obtain the existence of a positive $\omega$-periodic mild solution under the condition that the nonlinear function satisfies a linear growth condition concerning the growth exponent of the semigroup $T(t)$ $(t \geq 0)$. In the end, an example is given to illustrate the applicability of our abstract results.
\end{abstract}

MSC: $34 \mathrm{~K} 30 ; 47 \mathrm{H} 07 ; 47 \mathrm{H} 08$

Keywords: abstract evolution equation; positive periodic mild solutions; positive compact semigroup; the growth exponent of the semigroup; fixed point theorem

\section{Introduction}

Let $E$ be an ordered Banach space, whose positive cone $K$ is normal cone with normal constant $N$. In this paper, we discuss the existence of positive time periodic mild solutions for the evolution equation in the ordered Banach space $E$

$$
u^{\prime}(t)+A u(t)=f(t, u(t)), \quad t \in \mathbb{R},
$$

where $A: D(A) \subset E \rightarrow E$ is a closed linear operator and $-A$ generates a positive compact semigroup $T(t)(t \geq 0)$ in $E$, the nonlinear mapping $f: \mathbb{R} \times E \rightarrow E$ is continuous, and, for every $x \in E, f(t, x)$ is $\omega$-periodic in $t$.

It is well known that a variety of partial differential equations with time $t$, such as the reaction-diffusion equation, the heat equation, the wave equation, the telegraph equation and so on, can be classified as a nonlinear evolution equation (1.1) in some Banach space $E$. In the nonlinear evolution equation (1.1), $A$ corresponds to the linear partial differential operator with certain boundary conditions, $f$ corresponds to the nonlinear term.

It is noteworthy at this point that the problem concerning periodic solutions of partial differential equations has become an important area of investigation in recent years. Specially, the periodic problems of abstract evolution equations in the form of (1.1) have been considered by several authors; see [1-11].

D $2015 \mathrm{Li}$ and Li; licensee Springer. This is an Open Access article distributed under the terms of the Creative Commons Attribution License (http://creativecommons.org/licenses/by/4.0), which permits unrestricted use, distribution, and reproduction in any medium, provided the original work is properly credited. 
In [9], the existence and uniqueness for the periodic boundary value problem of the semilinear evolution equation in a Hilbert space $H$,

$$
\left\{\begin{array}{l}
u^{\prime}(t)+A u(t)=f(t, u(t)), \quad 0<t<\omega, \\
u(0)=u(\omega),
\end{array}\right.
$$

was studied, where $A: D(A) \subset H \rightarrow H$ is a positive definite self-adjoint operator and $A$ has compact resolvent. The author presented some spectral conditions for the nonlinearity $f(t, x)$ to guarantee the existence and uniqueness by applying the Schauder fixed point theorem and the contraction mapping principle. Specially, in [10], the author established the existence and uniqueness results of periodic solutions for the linear evolution equation corresponding to (1.1) and accurately estimated the spectral radius of periodic resolvent operator. With the aid of the estimation, under the assumption that the nonlinearity $f(t, x)$ satisfies some ordered conditions concerning the growth exponent of the semigroup $T(t)$ $(t \geq 0)$, the existence and uniqueness results of periodic mild solutions were obtained by applying the operator semigroup theorem and monotone iterative method.

Since in many practice models, only positive periodic solutions are significant, motivated by the papers mentioned above, we research the existence of positive $\omega$-periodic mild solutions for (1.1) in the positive cone $K$. In this paper, we will use a completely different method to prove the existence of positive $\omega$-periodic mild solutions for (1.1) under some new conditions by applying the Leray-Schauder fixed point theorem in an ordered Banach space $E$. More precisely, the nonlinear term satisfies a linear growth condition concerning the growth exponent of the semigroup $T(t)(t \geq 0)$ or the first eigenvalue of the operator $A$.

For the $C_{0}$-semigroup $T(t)(t \geq 0)$, there exist $M>0$ and $\gamma \in \mathbb{R}$ such that (see [12])

$$
\|T(t)\| \leq M e^{\gamma t}, \quad t \geq 0
$$

Let

$$
v_{0}=\inf \left\{\gamma \in \mathbb{R} \mid \text { there exists } M>0 \text { such that }\|T(t)\| \leq M e^{\gamma t}, \forall t \geq 0\right\} \text {, }
$$

then $v_{0}$ is called the growth exponent of the semigroup $T(t)(t \geq 0)$. Furthermore, $v_{0}$ can also be obtained by the following formula:

$$
v_{0}=\limsup _{t \rightarrow+\infty} \frac{\ln \|T(t)\|}{t}
$$

If $C_{0}$-semigroup $T(t)$ is continuous in the uniform operator topology for every $t>0$ in $E$, it is well known that $v_{0}$ can also be determined by $\sigma(A)$ (see [13])

$$
\nu_{0}=-\inf \{\operatorname{Re} \lambda \mid \lambda \in \sigma(A)\}
$$

where $-A$ is the infinitesimal generator of the $C_{0}$-semigroup $T(t)(t \geq 0)$. We know that $T(t)(t \geq 0)$ is continuous in the uniform operator topology for $t>0$ if $T(t)(t \geq 0)$ is a compact semigroup (see [1]). 
Our main results are as follows:

Theorem 1.1 Let $E$ be an ordered Banach space, whose positive cone $K$ is a normal cone, $A: D(A) \subset E \rightarrow E$ be a closed linear operator, and $-A$ generate an exponentially stable positive compact semigroup $T(t)(t \geq 0)$ in $E$, that is, $v_{0}<0$. Assume that $f: \mathbb{R} \times K \rightarrow K$ is continuous and $f(t, x)$ is $\omega$-periodic in $t$. If the following condition is satisfied:

(H1) there is a constant $c \in\left(0,\left|v_{0}\right|\right)$ and a function $h_{0} \in C_{\omega}(\mathbb{R}, K)$ such that

$$
f(t, x) \leq c x+h_{0}(t), \quad t \in \mathbb{R}, x \in K
$$

then (1.1) has at least one positive $\omega$-periodic mild solution $u$.

Theorem 1.2 Let $E$ be an ordered Banach space, whose positive cone $K$ is a normal cone, $A: D(A) \subset E \rightarrow E$ be a closed linear operator, and $-A$ generate an exponentially stable positive compact semigroup $T(t)(t \geq 0)$ in $E$. Assume that $f: \mathbb{R} \times K \rightarrow K$ is continuous and $f(t, x)$ is $\omega$-periodic in $t$. If the following condition is satisfied:

(H2) there is a constant $c \in\left(0,\left|v_{0}\right|\right)$ such that for $x, y \in K$ with $y \leq x$,

$$
f(t, x)-f(t, y) \leq c(x-y), \quad t \in \mathbb{R},
$$

then (1.1) has a unique positive $\omega$-periodic mild solution $u$.

Furthermore, we assume that the positive cone $K$ is a regeneration cone. By the characteristic of positive semigroups (see [14]), for sufficiently large $\lambda_{0}>-\inf \{\operatorname{Re} \lambda \mid \lambda \in \sigma(A)\}$, we see that $\lambda_{0} I+A$ has a positive bounded inverse operator $\left(\lambda_{0} I+A\right)^{-1}$. Since $\sigma(A) \neq \emptyset$, the spectral radius $r\left(\left(\lambda_{0} I+A\right)^{-1}\right)=\frac{1}{\operatorname{dist}\left(-\lambda_{0}, \sigma(A)\right)}>0$. By the famous Krein-Rutmann theorem, $A$ has the first eigenvalue $\lambda_{1}$, which has a positive eigenfunction $e_{1}$, and

$$
\lambda_{1}=\inf \{\operatorname{Re} \lambda \mid \lambda \in \sigma(A)\},
$$

that is, $v_{0}=-\lambda_{1}$. Hence, by Theorem 1.1 and Theorem 1.2, we have the following.

Corollary 1.3 Let E be an ordered Banach space, whose positive cone $K$ is a normal regeneration cone. -A generates an exponentially stable positive compact semigroup $T(t)(t \geq 0)$ in $E$. Assume that $f: \mathbb{R} \times K \rightarrow K$ is continuous and $f(t, x)$ is $\omega$-periodic in $t$. If the following condition is satisfied:

$(\mathrm{H} 1)^{\prime}$ there is a constant $c \in\left(0, \lambda_{1}\right)$ and a function $h_{0} \in C_{\omega}(\mathbb{R}, K)$ such that

$$
f(t, x) \leq c x+h_{0}(t), \quad t \in \mathbb{R}, x \in K,
$$

then (1.1) has at least one positive $\omega$-periodic mild solution $u$.

Corollary 1.4 Let E be an ordered Banach space, whose positive cone $K$ is a normal regeneration cone. - A generates an exponentially stable positive compact semigroup $T(t)(t \geq 0)$ in $E$. Assume that $f: \mathbb{R} \times K \rightarrow K$ is continuous and $f(t, x)$ is $\omega$-periodic in $t$. If the following condition is satisfied: 
$(\mathrm{H} 2)^{\prime}$ there is a constant $c \in\left(0, \lambda_{1}\right)$ such that for $x, y \in K$ with $y \leq x$,

$$
f(t, x)-f(t, y) \leq c(x-y), \quad t \in \mathbb{R}
$$

then (1.1) has a unique positive $\omega$-periodic mild solution $u$.

Remark 1.5 In Corollary 1.3 and Corollary 1.4, since $\lambda_{1}$ is the first eigenvalue of $A$, the condition $c<\lambda_{1}$ in $(\mathrm{H} 1)^{\prime}$ and $(\mathrm{H} 2)^{\prime}$ cannot be extended to $c \leq \lambda_{1}$. Otherwise, (1.1) does not always have a mild solution. For example, $f(t, x)=\lambda_{1} x$.

The paper is organized as follows. Section 2 provides the definitions and preliminary results to be used in theorems stated and proved in the paper. In Section 3, we apply the operator semigroup theory and the Leray-Schauder fixed point theorem to prove Theorem 1.1 and Theorem 1.2. In the last section, we give an example to illustrate the applicability of the abstract results.

\section{Preliminaries}

In this section, we introduce some notions, definitions, and preliminary facts which are used through this paper.

Let $J$ denote the infinite interval $[0,+\infty)$ and $h: J \rightarrow E$, consider the initial value problem of the linear evolution equation

$$
\left\{\begin{array}{l}
u^{\prime}(t)+A u(t)=h(t), \quad t \in J, \\
u(0)=x_{0}
\end{array}\right.
$$

It is well known [12], Chapter 4, Theorem 2.9, when $x_{0} \in D(A)$ and $h \in C^{1}(J, E)$, the initial value problem (2.1) has a unique classical solution $u \in C^{1}(J, E) \cap C\left(J, E_{1}\right)$ expressed by

$$
u(t)=T(t) x_{0}+\int_{0}^{t} T(t-s) h(s) d s
$$

where $E_{1}=D(A)$ is Banach space with the graph norm $\|\cdot\|_{1}=\|\cdot\|+\|A \cdot\|$. Generally, for $x_{0} \in E$ and $h \in C(J, E)$, the function $u$ given by (2.2) belongs to $C(J, E)$ and it is called a mild solution of the linear evolution equation (2.1).

Let $C_{\omega}(\mathbb{R}, E)$ denote the Banach space $\{u \in C(\mathbb{R}, E) \mid u(t+\omega)=u(t), t \in \mathbb{R}\}$ endowed with the maximum norm $\|u\|_{C}=\max _{t \in[0, \omega]}\|u(t)\|$. Evidently, $C_{\omega}(\mathbb{R}, E)$ is also an ordered Banach space with the partial order ' $\leq$ ' induced by the positive cone $K_{C}=\left\{u \in C_{\omega}(\mathbb{R}, E) \mid u(t) \geq\right.$ $\theta, t \in \mathbb{R}\}$ and $K_{C}$ is also normal with the normal constant $N$.

Given $h \in C_{\omega}(\mathbb{R}, E)$, for the following linear evolution equation corresponding to (1.1):

$$
u^{\prime}(t)+A u(t)=h(t), \quad t \in \mathbb{R}
$$

we have the following result.

Lemma 2.1 ([10]) If -A generates an exponentially stable positive $C_{0}$-semigroup $T(t)(t \geq$ $0)$ in $E$, then for $h \in C_{\omega}(\mathbb{R}, E)$, for the linear evolution equation (2.3) there exists a unique 
$\omega$-periodic mild solution $u$, which can be expressed by

$$
u(t)=(I-T(\omega))^{-1} \int_{t-\omega}^{t} T(t-s) h(s) d s:=(P h)(t),
$$

and the solution operator $P: C_{\omega}(\mathbb{R}, E) \rightarrow C_{\omega}(\mathbb{R}, E)$ is a positive bounded linear operator with the spectral radius $r(P) \leq \frac{1}{\left|v_{0}\right|}$.

Proof For any $v \in\left(0,\left|v_{0}\right|\right)$, there exists $M>0$ such that

$$
\|T(t)\| \leq M e^{-v t} \leq M, \quad t \geq 0 .
$$

In $E$, define the equivalent norm $|\cdot|$ by

$$
|x|=\sup _{t \geq 0}\left\|e^{v t} T(t) x\right\|
$$

then $\|x\| \leq|x| \leq M\|x\|$. By $|T(t)|$ we denote the norm of $T(t)$ in $(E,|\cdot|)$, then for $t \geq 0$, it is easy to obtain $|T(t)|<e^{-v t}$. Hence, $(I-T(\omega))$ has a bounded inverse operator

$$
(I-T(\omega))^{-1}=\sum_{n=0}^{\infty} T(n \omega)
$$

and its norm satisfies

$$
\left|(I-T(\omega))^{-1}\right| \leq \frac{1}{1-|T(\omega)|} \leq \frac{1}{1-e^{-\nu \omega}} .
$$

Set

$$
x_{0}=(I-T(\omega))^{-1} \int_{0}^{\omega} T(t-s) h(s) d s:=B h,
$$

then the mild solution $u(t)$ of the linear initial value problem (2.1) given by (2.2) satisfies the periodic boundary condition $u(0)=u(\omega)=x_{0}$. For $t \in \mathbb{R}^{+}$, by (2.2) and the properties of the semigroup $T(t)(t \geq 0)$, we have

$$
\begin{aligned}
u(t+\omega) & =T(t+\omega) u(0)+\int_{0}^{t+\omega} T(t+\omega-s) h(s) d s \\
& =T(t)\left(T(\omega) u(0)+\int_{0}^{\omega} T(\omega-s) h(s) d s\right)+\int_{0}^{t} T(t-s) h(s-\omega) d s \\
& =T(t) u(0)+\int_{0}^{t} T(t-s) h(s) d s=u(t) .
\end{aligned}
$$

Therefore, the $\omega$-periodic extension of $u$ on $\mathbb{R}$ is a unique $\omega$-periodic mild solution of (2.3). By (2.2) and (2.8), the $\omega$-periodic mild solution can be expressed by

$$
\begin{aligned}
u(t) & =T(t) B(h)+\int_{0}^{t} T(t-s) h(s) d s \\
& =(I-T(\omega))^{-1} \int_{t-\omega}^{t} T(t-s) h(s) d s:=(P h)(t) .
\end{aligned}
$$


Evidently, by the positivity of the semigroup $T(t)(t \geq 0)$, we can see that $P: C_{\omega}(\mathbb{R}, E) \rightarrow$ $C_{\omega}(\mathbb{R}, E)$ is a positive bounded linear operator. By (2.7) and (2.9), we have

$$
\begin{aligned}
|(P h)(t)| & \leq\left|(I-T(\omega))^{-1}\right| \int_{t-\omega}^{t}|T(t-s) h(s)| d s \\
& \leq \frac{1}{1-e^{-\nu \omega}} \int_{t-\omega}^{t} e^{-v(t-s)}|h|_{C} d s \leq \frac{1}{v}|h|_{C},
\end{aligned}
$$

which implies that $|P| \leq \frac{1}{v}$. Therefore, $r(P) \leq|P| \leq \frac{1}{v}$. Hence, by the arbitrariness of $v \in$ $\left(0,\left|v_{0}\right|\right)$, we have $r(P) \leq \frac{1}{\left|v_{0}\right|}$. This completes the proof of Lemma 2.1.

In the proof of our main results, we need the following fixed point theorem.

Lemma 2.2 (Leray-Schauder fixed point theorem [15]) Let $\Omega$ be a convex subset of Banach space $E$ with $\theta \in \Omega$, and let $Q: \Omega \rightarrow \Omega$ be a compact operator. If the set $\{u \in \Omega \mid u=\eta Q u, 0<$ $\eta<1\}$ is bounded, then $Q$ has a fixed point in $\Omega$.

\section{Proof of the main results}

Proof of Theorem 1.1 Evidently, the normal cone $K_{C}$ is a convex subset of the Banach space $C_{\omega}(\mathbb{R}, E)$ and $\theta \in K_{C}$. Consider the operator $Q$ defined by

$$
Q u=(P \circ F)(u),
$$

where

$$
F(u):=f(\cdot, u(\cdot)), \quad u \in K_{C} .
$$

From the positivity of semigroup of $T(t)(t \geq 0)$ and the conditions of Theorem 1.1, it is easy to see that $Q: K_{C} \rightarrow K_{C}$ is well defined. It is clear that

$$
(Q u)(t)=(I-T(\omega))^{-1} \int_{t-\omega}^{t} T(t-s) f(s, u(s)) d s, \quad t \in \mathbb{R} .
$$

By the definition of $P$, the positive $\omega$-periodic mild solution of (1.1) is equivalent to the fixed point of the operator $Q$. In the following, we will prove $Q$ has a fixed point by applying the famous Leray-Schauder fixed point theorem.

At first, we prove that $Q$ is continuous on $K_{C}$. To this end, let $\left\{u_{m}\right\} \subset K_{C}$ be a sequence such that $u_{m} \rightarrow u \in K_{C}$ as $m \rightarrow \infty$, so for every $t \in \mathbb{R}, \lim _{m \rightarrow \infty} u_{m}(t)=u(t)$. Since $f$ : $\mathbb{R} \times K \rightarrow K$ is continuous, for every $t \in \mathbb{R}$, we get

$$
f\left(t, u_{m}(t)\right) \rightarrow f(t, u(t)), \quad m \rightarrow \infty
$$

By (3.3) and the Lebesgue dominated convergence theorem, for every $t \in \mathbb{R}$, we have

$$
\begin{aligned}
& \left\|\left(Q u_{m}\right)(t)-(Q u)(t)\right\| \\
& \quad=\left\|(I-T(\omega))^{-1}\left(\int_{t-\omega}^{t} T(t-s) f\left(s, u_{m}(s)\right) d s-\int_{t-\omega}^{t} T(t-s) f(s, u(s)) d s\right)\right\|
\end{aligned}
$$




$$
\begin{aligned}
& \leq\left\|(I-T(\omega))^{-1}\right\| \cdot \int_{t-\omega}^{t}\|T(t-s)\| \cdot\left\|f\left(s, u_{m}(s)\right)-f(s, u(s))\right\| d s \\
& \leq C M \cdot \int_{t-\omega}^{t}\left\|f\left(s, u_{m}(s)\right)-f(s, u(s))\right\| d s,
\end{aligned}
$$

where $C=\left\|(I-T(\omega))^{-1}\right\|$. Therefore, we can conclude that

$$
\left\|Q u_{m}-Q u\right\| \rightarrow 0, \quad m \rightarrow \infty
$$

Thus, $Q: K_{C} \rightarrow K_{C}$ is continuous.

Subsequently, we show that $Q$ maps every bounded set in $K_{C}$ into a bounded set. For any $R>0$, let

$$
\Omega_{R}:=\left\{u \in K_{C} \mid\|u\|_{C} \leq R\right\}
$$

For each $u \in \Omega_{R}$, from the continuity of $f$, we know that there exists $M_{1}>0$ such that

$$
\|f(t, u(t))\| \leq M_{1}, \quad t \in \mathbb{R}
$$

hence, we get

$$
\begin{aligned}
\|(Q u)(t)\| & =\left\|(I-T(\omega))^{-1} \int_{t-\omega}^{t} T(t-s) f(s, u(s)) d s\right\| \\
& \leq\left\|(I-T(\omega))^{-1}\right\| \int_{t-\omega}^{t}\|T(t-s)\| \cdot\|f(s, u(s))\| d s \\
& \leq C M \int_{t-\omega}^{t} M_{1} d s \\
& \leq C M M_{1} \omega:=\bar{R} .
\end{aligned}
$$

Therefore, $Q\left(\Omega_{R}\right)$ is bounded.

Next, we demonstrate that $Q\left(\Omega_{R}\right)$ is equicontinuous. For every $u \in \Omega_{R}$, by the periodicity of $u$, we only consider it on $[0, \omega]$. Set $0 \leq t_{1}<t_{2} \leq \omega$, we get

$$
\begin{aligned}
Q u\left(t_{2}\right)-Q u\left(t_{1}\right) & \\
= & (I-T(\omega))^{-1} \int_{t_{2}-\omega}^{t_{2}} T\left(t_{2}-s\right) f(s, u(s)) d s \\
& -(I-T(\omega))^{-1} \int_{t_{1}-\omega}^{t_{1}} T\left(t_{1}-s\right) f(s, u(s)) d s \\
= & (I-T(\omega))^{-1} \int_{t_{2}-\omega}^{t_{1}}\left(T\left(t_{2}-s\right)-T\left(t_{1}-s\right)\right) f(s, u(s)) d s \\
& -(I-T(\omega))^{-1} \int_{t_{1}-\omega}^{t_{2}-\omega} T\left(t_{1}-s\right) f(s, u(s)) d s \\
& +(I-T(\omega))^{-1} \int_{t_{1}}^{t_{2}} T\left(t_{2}-s\right) f(s, u(s)) d s \\
:= & I_{1}+I_{2}+I_{3},
\end{aligned}
$$


where

$$
\begin{aligned}
& I_{1}=(I-T(\omega))^{-1} \int_{t_{2}-\omega}^{t_{1}}\left(T\left(t_{2}-s\right)-T\left(t_{1}-s\right)\right) f(s, u(s)) d s, \\
& I_{2}=-(I-T(\omega))^{-1} \int_{t_{1}-\omega}^{t_{2}-\omega} T\left(t_{1}-s\right) f(s, u(s)) d s, \\
& I_{3}=(I-T(\omega))^{-1} \int_{t_{1}}^{t_{2}} T\left(t_{2}-s\right) f(s, u(s)) d s .
\end{aligned}
$$

It is clear that

$$
\left\|Q u\left(t_{2}\right)-Q u\left(t_{1}\right)\right\| \leq\left\|I_{1}\right\|+\left\|I_{2}\right\|+\left\|I_{3}\right\| .
$$

Now, we only need to check that the $\left\|I_{i}\right\|$ tend to 0 independently of $u \in \Omega_{R}$ when $t_{2}-t_{1} \rightarrow 0, i=1,2,3$. From the definition of the $I_{i}$, we can easily see

$$
\begin{aligned}
\left\|I_{1}\right\| & \leq C \cdot \int_{t_{2}-\omega}^{t_{1}}\left\|\left(T\left(t_{2}-s\right)-T\left(t_{1}-s\right)\right)\right\| \cdot\|f(s, u(s))\| d s \\
& \leq C M_{1} \int_{t_{2}-\omega}^{t_{1}}\left\|\left(T\left(t_{2}-s\right)-T\left(t_{1}-s\right)\right)\right\| d s \\
& \rightarrow 0, \quad \text { as } t_{2}-t_{1} \rightarrow 0, \\
\left\|I_{2}\right\| & \leq C \cdot \int_{t_{1}-\omega}^{t_{2}-\omega}\left\|\left(T\left(t_{1}-s\right)\right)\right\| \cdot\|f(s, u(s))\| d s \\
& \leq C M M_{1}\left(t_{2}-t_{1}\right) \\
& \rightarrow 0, \quad \text { as } t_{2}-t_{1} \rightarrow 0, \\
\left\|I_{3}\right\| & \leq C \cdot \int_{t_{1}}^{t_{2}}\left\|\left(T\left(t_{2}-s\right)\right)\right\| \cdot\|f(s, u(s))\| d s \\
& \leq C M M_{1}\left(t_{2}-t_{1}\right) d s \\
& \rightarrow 0, \quad \text { as } t_{2}-t_{1} \rightarrow 0 .
\end{aligned}
$$

As a result, $\left\|Q u\left(t_{2}\right)-Q u\left(t_{1}\right)\right\|$ tends to 0 independently of $u \in \Omega_{R}$ as $t_{2}-t_{1} \rightarrow 0$, which means that $Q\left(\Omega_{R}\right)$ is equicontinuous.

Now, we prove that $\left(Q \Omega_{R}\right)(t)$ is relatively compact in $K$ for all $t \in \mathbb{R}$. To this end, we define a set $\left(Q_{\varepsilon} \Omega_{R}\right)(t)$ by

$$
\left(Q_{\varepsilon} \Omega_{R}\right)(t):=\left\{\left(Q_{\varepsilon} u\right)(t) \mid u \in \Omega_{R}, 0<\varepsilon<\omega, t \in \mathbb{R}\right\},
$$

where

$$
\begin{aligned}
\left(Q_{\varepsilon} u\right)(t) & =(I-T(\omega))^{-1} \int_{t-\omega}^{t-\varepsilon} T(t-s) f(s, u(s)) d s \\
& =T(\varepsilon)(I-T(\omega))^{-1} \int_{t-\omega}^{t-\varepsilon} T(t-s-\varepsilon) f(s, u(s)) d s .
\end{aligned}
$$


Then the set $\left(Q_{\varepsilon} \Omega_{R}\right)(t)$ is relatively compact in $K$ since the operator $T(\varepsilon)$ is compact in $K$. For any $u \in \Omega_{R}$ and $t \in \mathbb{R}$, from the following inequality:

$$
\begin{aligned}
& \left\|Q u(t)-Q_{\varepsilon} u(t)\right\| \\
& \quad=\left\|(I-T(\omega))^{-1}\left(\int_{t-\omega}^{t} T(t-s) f(s, u(s)) d s-\int_{t-\omega}^{t-\varepsilon} T(t-s) f(s, u(s))\right) d s\right\| \\
& \quad \leq C \int_{t-\varepsilon}^{t}\|T(t-s) f(s, u(s))\| d s \\
& \quad \leq C M M_{1} \varepsilon,
\end{aligned}
$$

one see that the set $\left(Q \Omega_{R}\right)(t)$ is relatively compact in $K$ for all $t \in \mathbb{R}$.

Thus, the Arzela-Ascoli theorem guarantees that $Q: K_{C} \rightarrow K_{C}$ is a compact operator.

Finally, we prove the set $\Lambda(Q):=\left\{u \in K_{C} \mid u=\eta Q u, \forall 0<\eta<1\right\}$ is bounded. For every $u \in K_{C}$, by (3.2) and the condition (H1), we have

$$
\theta \leq F(u)(t)=f(t, u(t)) \leq c u(t)+h_{0}(t), \quad t \in \mathbb{R} .
$$

Let $u \in \Lambda(Q)$, then there is a constant $\eta \in(0,1)$ such that $u=\eta Q u$. Therefore, by the definition of $Q$, Lemma 2.1, and (3.12), we have

$$
\begin{aligned}
\theta & \leq u(t)=\eta(Q u)(t)<(Q u)(t) \\
& =P \circ F(u)(t) \leq P\left(c u(t)+h_{0}(t)\right) \\
& =c P u(t)+P h_{0}(t)<c(P \circ Q u)(t)+P h_{0}(t) \\
& \leq c P\left(c P u(t)+P h_{0}(t)\right)+P h_{0}(t) \\
& =c^{2} P^{2} u(t)+c P^{2} h_{0}(t)+P h_{0}(t),
\end{aligned}
$$

inductively, we can see

$$
u(t) \leq c^{n} P^{n} u(t)+\mathcal{P} h_{0}(t), \quad n=1,2, \ldots,
$$

where $\mathcal{P}=c^{n-1} P^{n}+c^{n-2} P^{n-1}+\cdots+c P^{2}+P$ is a bounded linear operator, and there exists a constant $M_{2}>0$ such that $\|\mathcal{P}\| \leq M_{2}$. Hence, by the normality of the cone $K_{C}$, we can see

$$
\|u\|_{C}<c^{n} N\left\|P^{n}\right\| \cdot\|u\|_{C}+M_{2}\left\|h_{0}\right\|_{C} .
$$

From the spectral radius of the Gelfand formula $\lim _{n \rightarrow \infty} \sqrt[n]{\left\|P^{n}\right\|}=r(P)=\frac{1}{\left|\nu_{0}\right|}$, and the condition (H1), when $n$ is large enough, we get $c^{n}\left\|P^{n}\right\|<\frac{1}{N}$, then

$$
\|u\|_{C}<\frac{M_{2}\left\|h_{0}\right\|_{C}}{1-N c^{n}\left\|P^{n}\right\|}
$$

which implies that $\Lambda(Q)$ is bounded. By the Leray-Schauder fixed point theorem of a compact operator, the operator $Q$ has at least one fixed point $u$ in $K_{C}$, which is a positive $\omega$ periodic mild solution of (1.1). This completes the proof of Theorem 1.1. 
Proof of Theorem 1.2 From the condition (H2), it is easy to see that the condition (H1) holds. Hence by Theorem 1.1, (1.1) has positive $\omega$-periodic mild solutions. Let $u_{1}, u_{2} \in K_{C}$ be the positive $\omega$-periodic solutions of (1.1), then they are the fixed points of the operator $Q=P \circ F$. Let us assume $u_{1} \leq u_{2}$, by the definition of $F$ and the condition (H2), we see that $F\left(u_{2}\right)(t)-F\left(u_{1}\right)(t) \leq c\left(u_{2}(t)-u_{1}(t)\right)$. So by Lemma 2.1, we obtain

$$
\begin{aligned}
\theta & \leq u_{2}(t)-u_{1}(t)=\left(Q u_{2}\right)(t)-\left(Q u_{1}\right)(t)=P\left(\left(F u_{2}\right)(t)-\left(F u_{1}\right)(t)\right) \\
& \leq c P\left(u_{2}(t)-u_{1}(t)\right) \leq \cdots \leq c^{n} P^{n}\left(u_{2}(t)-u_{1}(t)\right) .
\end{aligned}
$$

By the normality of the cone $K_{C}$, we can see that

$$
\left\|u_{2}-u_{1}\right\|_{C} \leq N c^{n}\left\|P^{n}\right\| \cdot\left\|u_{2}-u_{1}\right\|_{C} .
$$

From the proof of Theorem 1.1, when $n$ is large enough, $N c^{n}\left\|P^{n}\right\|<1$, so $\left\|u_{2}-u_{1}\right\|_{C}=0$, it follows that $u_{2} \equiv u_{1}$. Thus, (1.1) has only one positive $\omega$-periodic mild solution.

\section{Application}

In this section, we present one example, which indicates how our abstract results can be applied to concrete problems.

Let $\Omega \in \mathbb{R}^{N}$ be a bounded domain with a sufficiently smooth boundary $\partial \Omega$. Let

$$
A(x, D) u=-\sum_{i, j=1}^{N} a_{i j}(x) D_{i} D_{j} u+\sum_{j=1}^{N} a_{j}(x) D_{j} u+a_{0}(x) u
$$

be a uniformly elliptic differential operator in $\bar{\Omega}$, whose coefficients $a_{i j}(x), a_{j}(x)(i, j=$ $1, \ldots, N)$ and $a_{0}(x)$ are Hölder-continuous on $\bar{\Omega}$ and $a_{0}(x) \geq 0$. We let $B=B(x, D)$ be a boundary operator on $\partial \Omega$ of the form

$$
B u:=b_{0}(x) u+\delta \frac{\partial u}{\partial \beta},
$$

where either $\delta=0$ and $b_{0}(x) \equiv 1$ (Dirichlet boundary operator), or $\delta=1$ and $b_{0}(x) \geq 0$ (regular oblique derivative boundary operator; at this point, we further assume that $a_{0}(x) \not \equiv 0$ or $\left.b_{0}(x) \not \equiv 0\right), \beta$ is an outward pointing, nowhere tangent vector field on $\partial \Omega$.

Let $\lambda_{1}$ be the first eigenvalue of elliptic operator $A(x, D)$ under the boundary condition $B u=0$. It is well known ([16], Theorem 1.16) that $\lambda_{1}>0$.

Under the above assumptions, we discuss the existence and uniqueness of positive time $\omega$-periodic solutions of the semilinear parabolic boundary value problem

$$
\left\{\begin{array}{l}
\frac{\partial}{\partial t} u(x, t)+A(x, D) u(x, t)=g(x, t, u(x, t)), \quad(x, t) \in \Omega \times \mathbb{R} \\
B u=0, \quad x \in \partial \Omega
\end{array}\right.
$$

where $g: \bar{\Omega} \times \mathbb{R} \times \mathbb{R} \rightarrow \mathbb{R}$ is a local Hölder-continuous function which is $\omega$-periodic in $t$.

Let $E=L^{p}(\Omega)(p>1), K=\{u \in E \mid u(x) \geq 0$ a.e. $x \in \Omega\}$, then $E$ is an ordered Banach space, whose positive cone $K$ is a normal regeneration cone. Define an operator $A: D(A) \subset$ $E \rightarrow E$ by

$$
D(A)=\left\{u \in W^{2, p}(\Omega) \mid B(x, D) u=0, x \in \partial \Omega\right\}, \quad A u=A(x, D) u .
$$


If $a_{0}(x) \geq 0$, then $-A$ generates an exponentially stable analytic semigroup $T_{p}(t)(t \geq 0)$ in $E$ (see [17]). By the maximum principle of elliptic operators, we know that $(\lambda I+A)$ has a positive bounded inverse operator $(\lambda I+A)^{-1}$ for $\lambda>0$, hence $T_{p}(t)(t \geq 0)$ is a positive semigroup (see [14]). From the operator $A$ has compact resolvent in $L^{p}(\Omega)$, we obtain $T_{p}(t)$ $(t \geq 0)$ is also a compact semigroup (see [12]).

Therefore, by Corollary 1.3, we have the following result.

Theorem 4.1 Assume that $g: \bar{\Omega} \times \mathbb{R} \times \mathbb{R} \rightarrow \mathbb{R}$ is a local Hölder-continuous function which is $\omega$-periodic in $t$ and satisfies $g(x, t, u) \geq 0$ for $(x, t, u) \in\left(\bar{\Omega} \times \mathbb{R} \times \mathbb{R}^{+}\right)$. If the following condition holds:

(H3) there is a constant $c \in\left(0, \lambda_{1}\right)$ and a function $h_{0} \in C_{\omega}(\Omega \times \mathbb{R})$ satisfying $h_{0}(x, t) \geq 0$ such that

$$
g(x, t, u) \leq c u+h_{0}(x, t), \quad(x, t) \in \Omega \times \mathbb{R}, u \geq 0,
$$

then the parabolic boundary value problem (4.3) has at least one positive $\omega$-periodic solution $u \in C^{2,1}(\bar{\Omega} \times \mathbb{R})$.

Proof Let $u(t)=u(\cdot, t), f(t, u(t))=g(\cdot, t, u(\cdot, t))$, then the parabolic boundary value problem (4.3) can be reformulated as the abstract evolution (1.1) in $E$. It is easy to see that the conditions of Corollary 1.3 are satisfied. By Corollary 1.3, the parabolic boundary value problem (4.3) has a time positive $\omega$-periodic mild solution $u \in C_{\omega}(\mathbb{R}, E)$. By the analyticity of the semigroup $T_{p}(t)(t \geq 0)$ and the regularization method used in [17], we can see that $u \in C^{2,1}(\bar{\Omega} \times \mathbb{R})$ is a classical time $\omega$-periodic solution of the problem (4.3). This completes the proof of the theorem.

From Corollary 1.4 and Theorem 4.1, we obtain the uniqueness result.

Theorem 4.2 Assume that $g: \bar{\Omega} \times \mathbb{R} \times \mathbb{R} \rightarrow \mathbb{R}$ is a local Hölder-continuous function which is $\omega$-periodic in $t$ and satisfies $g(x, t, u) \geq 0$ for $(x, t, u) \in\left(\bar{\Omega} \times \mathbb{R} \times \mathbb{R}^{+}\right)$. If the following condition holds:

(H4) there is a constant $c \in\left(0, \lambda_{1}\right)$ such that for $0<v<w$

$$
g(x, t, w)-g(x, t, v) \leq c(w-v), \quad(x, t) \in \Omega \times \mathbb{R},
$$

then the parabolic boundary value problem (4.3) has a unique positive $\omega$-periodic solution $u \in C^{2,1}(\bar{\Omega} \times \mathbb{R})$. 


\section{References}

1. Hino, Y, Murakami, S: Periodic solutions of linear Voltera systems. In: Differential Equations. Lect. Notes Pure Appl. Math., vol. 118, pp. 319-326. Dekker, New York (1987)

2. Vrabie, I: Periodic solutions for nonlinear evolution equations in a Banach space. Proc. Am. Math. Soc. 109(3), 653-661 (1990)

3. Liu, J: Bounded and periodic solutions of differential equations in Banach space. Appl. Math. Comput. 65, 141-150 (1994)

4. Liu, J: Bounded and periodic solutions of semi-linear evolution equations. Dyn. Syst. Appl. 4, 341-350 (1995)

5. Li, Y: Periodic solutions of semilinear evolution equations in Banach spaces. Acta Math. Sin. 41, 629-636 (1998) (in Chinese)

6. Li, Y, Cong, F, Lin, Z, Lin, W: Periodic solutions for evolution equations. Nonlinear Anal. 36, 275-293 (1999)

7. Akrid, T, Maniar, L, Ouhinou, A: Periodic solutions for some nonautonomous semilinear boundary evolution equations. Nonlinear Anal. 71, 1000-1011 (2009)

8. Zitane, M, Bensoida, C: Massera problem for non-autonomous retarded differential equations. J. Math. Anal. Appl. 402, 453-462 (2013)

9. $L i, Y$ : Existence and uniqueness of periodic solution for a class of semilinear evolution equations. J. Math. Anal. Appl. $349,226-234$ (2009)

10. Li, Y: Existence and uniqueness of positive periodic solution for abstract semilinear evolution equations. J. Syst. Sci. Math. Sci. 25, 720-728 (2005) (in Chinese)

11. Li, Y: Existence and asymptotic stability of periodic solution for evolution equations with delays. J. Funct. Anal. 261, 1309-1324 (2011)

12. Pazy, A: Semigroup of Linear Operators and Applications to Partial Differential Equations. Applied Mathematical Sciences, vol. 44. Springer, New York (1983)

13. Triggiani, R: On the stabilizability problem in Banach space. J. Math. Anal. Appl. 52, 383-403 (1975)

14. Li, Y: The positive solutions of abstract semilinear evolution equations and their applications. Acta Math. Sin. 39 666-672 (1996) (in Chinese)

15. Deimling, K: Nonlinear Functional Analysis. Springer, New York (1985)

16. Amann, $\mathrm{H}$ : Nonlinear operators in ordered Banach spaces and some applications to nonlinear boundary value problem. In: Nonlinear Operators and the Calculus of Variations. Lecture Notes in Mathematics, pp. 1-55. Springer, Berlin (1976)

17. Amann, H: Periodic solutions of semilinear parabolic equations. In: Cesari, L, Kannan, R, Weinberger, R (eds.) Nonlinear Analysis: A Collection of Papers in Honor of Erich H Rothe, pp. 1-29. Academic Press, New York (1978)

\section{Submit your manuscript to a SpringerOpen ${ }^{\odot}$ journal and benefit from:}

$\checkmark$ Convenient online submission

- Rigorous peer review

- Immediate publication on acceptance

- Open access: articles freely available online

- High visibility within the field

- Retaining the copyright to your article 